\title{
DHEA protects mitochondria against dual modes of apoptosis and necroptosis in human granulosa $\mathrm{HO} 23$ cells
}

\author{
Kuan-Hao Tsui ${ }^{1,2,3}$, Peng-Hui Wang $4,5,6,7,8$, Li-Te Lin ${ }^{1,2,4}$ and Chia-Jung Li ${ }^{9}$ \\ ${ }^{1}$ Department of Obstetrics and Gynecology, Kaohsiung Veterans General Hospital, Kaohsiung, Taiwan, ${ }^{2}$ Department \\ of Obstetrics and Gynecology, National Yang-Ming University School of Medicine, Taipei, Taiwan, ${ }^{3}$ Department of \\ Pharmacy and Master Program, College of Pharmacy and Health Care, Tajen University, Pingtung County, Taiwan, \\ ${ }^{4}$ Department of Biological Science, National Sun Yat-sen University, Kaohsiung, Taiwan, ${ }^{5}$ Division of Gynecology, \\ Department of Obstetrics and Gynecology, Taipei Veterans General Hospital, Taipei, Taiwan, ${ }^{6}$ Department of \\ Obstetrics and Gynecology, National Yang-Ming University Hospital, Ilan, Taiwan, ${ }^{7}$ Immunology Center, Taipei \\ Veterans General Hospital, Taipei, Taiwan, ${ }^{8}$ Department of Medical Research, China Medical University Hospital, \\ Taichung, Taiwan and ${ }^{9}$ Research Assistant Center, Show Chwan Health Memorial Hospital, Changhua, Taiwan
}

Correspondence should be addressed to K-H Tsui; Email: khtsui60@gmail.com

\begin{abstract}
Because ovarian granulosa cells are essential for oocyte maturation and development, we validated human granulosa $\mathrm{HO} 23$ cells to evaluate the ability of the DHEA to prevent cell death after starvation. The present study was aimed to investigate whether DHEA could protect against starvation-induced apoptosis and necroptosis in human oocyte granulosa HO23 cells. The starvation was induced by treatment of serum-free (SF) medium for $4 \mathrm{~h}$ in vitro. Starvation-induced mitochondrial depolarization, cytochrome c release and caspase-3 activation were largely prevented by DHEA in HO23 cells. We found that treatment with DHEA can restore starvation-induced reactive oxygen species (ROS) generation and mitochondrial membrane potential imbalance. In addition, treatment of DHEA prevents cell death via upregulation of cytochrome $c$ and downregulation of BAX in mitochondria. Most importantly, DHEA is ameliorated to mitochondrial function mediated through the decrease in mitochondrial ROS, maintained mitochondrial morphology, and enhancing the ability of cell proliferation and ROS scavenging. Our present data strongly indicate that DHEA reduces programmed cell death (apoptosis and necroptosis) in granulosa $\mathrm{HO} 23$ cells through multiple interactions with the mitochondrion-dependent programmed cell death pathway. Taken together, our data suggest that the presence of DHEA could be beneficial to protect human oocyte granulosa $\mathrm{HO} 23$ cells under in vitro culture conditions during various assisted reproductive technology (ART) programs.

Free Chinese abstract: A Chinese translation of this abstract is freely available at http://www.reproduction-online.org/ content/154/2/101/suppl/DC1.

Reproduction (2017) $\mathbf{1 5 4} 101-110$
\end{abstract}

\section{Introduction}

Oocytes were protected and nurtured from surrounding somatic cells, including cumulus cells (CCs) and granulosa cells (GCs) (Fragouli et al. 2014). Previous studies indicate that gene expression in GCs can serve as biomarkers for oocyte or embryo quality and pregnancy outcomes (Cillo et al. 2007, Anderson et al. 2009, Wathlet et al. 2012, Li et al. 2015b). Improvement in poor ovarian reserve (PORs) is one of the pivotal steps in assisted reproductive technology (ART). Dehydroepiandrosterone (DHEA) has been reported to improve oocyte quality, fertilization rate, embryo quality and pregnancy rate in diminished ovarian reserve patients. DHEA, a precursor to estradiol and testosterone, is produced by the zona reticularis of the adrenal glands and by ovarian theca cells (Labrie 2015,
Lin et al. 2015). An updated Cochrane review concluded that pretreatment with DHEA may be associated with improved live birth rates in PORs undergoing IVF (Nagels et al. 2015). However, the mechanism of improved IVF outcomes in PORs following DHEA treatment remains unclear.

Recent research suggests that ovarian aging may represent a continuum of premature ovarian senescence. Whether DHEA objectively improves ovarian reserve is, however, not yet fully elucidated. Just how DHEA affects the oocyte/embryo quality, granulosa cell proliferation and differentiation is not yet clear.

Our previous data displayed that the BCL2/BAX ratio of oocyte significantly increased in PORs after DHEA supplementation, indicating the anti-apoptotic effect on oocyte following DHEA treatment (Tsui et al. 
2014). Apoptosis plays a critical role on oogenesis, folliculogenesis, oocyte loss, selection, atresia and luteogenesis (Hussein 2005). Several studies indicate that apoptosis of GCs is associated with impaired oocyte maturation, fertilization, embryo growth and pregnancy outcome (Lee et al. 2001, Host et al. 2002, Corn et al. 2005). Mitochondria play an important role on the intrinsic apoptosis pathway mediated by the BCL2 family (Xiong et al. 2014). When stress stimuli are transduced to mitochondria, $\mathrm{BAX}$ and $\mathrm{BAK}$, proapoptotic members of the BCL2 family, increase the mitochondrial membrane permeability to proteins such as cytochrome $c$, leading to caspase cascade activation (Roy et al. 2014). Mitochondria are also involved in oocyte growth and embryo development (RamalhoSantos et al. 2009, Van Blerkom 2011). Dysfunctional mitochondria can contribute to poor oocyte maturation, fertilization and embryo formation (Santos et al. 2006, Zeng et al. 2007, Lee et al. 2014).

Currently, necroptosis is found to be a new type of programmed cell death that is different with apoptosis and necrosis. Recently, necroptosis has been found to be involved in some pathological conditions. It not only contributes to ischemic injury in heart and kidney (Smith et al. 2007, Linkermann et al. 2012), but also accelerates ovarian follicle necroptosis during aging (Blohberger et al. 2015).

Based on our previous study (Tsui et al. 2014) and the numerous studies discussed thus far, we hypothesized that DHEA may improve reproductive outcomes through increased mitochondrial function and decreased apoptosis of CCs. To test this hypothesis, we used human oocyte granulosa $\mathrm{HO} 23$ cells, an immortalized cell culture line. Furthermore, $\mathrm{HO} 23$ cells are very similar, if not identical, to non-pathological primary CCs with respect to their metabolizing activity of steroid hormones and their responsiveness to steroid hormones (Hosokawa et al. 1998, Lan et al. 2013). In this study, we tested DHEA to find out the optimized concentrations of each that produced maximum protection for $\mathrm{HO} 23$ cells suffering from starvation. However, the molecular mechanism by which treatment of DHEA in helping GCs to counter programmed cell death is still not fully elucidated. Then, we explored the major signal transduction pathways and multiple mechanisms in this process. The present investigation tested the hypothesis that DHEA could protect GCs from apoptosis and necroptosis by mitochondrial protection.

\section{Materials and methods \\ Cell culture and treatment}

The human GC cell line HO23 was established by triple transfection of primary human GCs with simian virus 40 DNA, Ha-ras oncogene and a TS mutant of p53 (p53 Val135) as described previously (Hosokawa et al. 1998). The cells were cultured in a medium containing Dulbecco's Modified Eagle Medium/Nutrient Mixture F-12 (DMEM/F12; GIBCO Invitrogen Co.) with $10 \% \mathrm{FBS}$ (GIBCO Invitrogen Co.) at $37^{\circ} \mathrm{C}$ in a humidified incubator containing $5 \% \mathrm{CO}_{2}$ and $95 \%$ air. The cells cultured in a complete medium (10\% FBS) were used as the control. For starvation and the DHEA experiment, the cells were pretreated with $0 \%$ FBS for $4 \mathrm{~h}$, followed by incubation in a medium without FBS and various concentrations of DHEA solution $(1.0 \mathrm{mg} / \mathrm{mL}$ in methanol, Sigma) for $20 \mathrm{~h}$.

\section{Cell proliferation assay}

The cell viability was analyzed using the cell counting kit-8 (CCK-8) that detected the metabolic activity of the cells. At the end of the various treatments, $10 \mu \mathrm{L}$ of the CCK-8 reagent was added to each well, and the cells were then incubated at $37^{\circ} \mathrm{C}$ for $4 \mathrm{~h}$. Absorbance was recorded using an ELISA microplate reader at $450 \mathrm{~nm}$.

\section{Cell death measurement}

Cell survival was assessed by labeling cells with propidium iodide (PI). Briefly, the cells were washed twice with PBS, re-suspended in a binding buffer and stained with $1 \mu \mathrm{g} / \mathrm{mL}$ of a Pl solution (Sigma) for $15 \mathrm{~min}$ at room temperature in the dark. After incubation, the cells were analyzed using flow cytometry. Cell fluorescence was measured with a FACScan flow cytometer (BD Bioscience, CA, USA). Side scatter and forward scatter were determined simultaneously, and all data were collected with FACScan research software. The data were analyzed with FlowJo software (Treestar Inc., CA, USA).

\section{Cell morphology staining}

Cell morphology was studied by Hoechst 33258 and PI double staining for live-cell images. After treatment, cells were stained Hoechst 33258 and PI solution at room temperature for $10 \mathrm{~min}$. Cells were washed twice with PBS for analysis by inverted fluorescence microscope (Carl Zeiss Axiovert M200).

\section{Cellular production of ROS}

Intracellular ROS production of ROS, namely hydrogen peroxide was measured using DCFDA (Enzo Life Sciences Inc.). Cells from each experimental condition were stained with DCFDA $(2 \mu \mathrm{M})$ at $37^{\circ} \mathrm{C}$ for $30 \mathrm{~min}$. ROS production of cells was subjected to evaluation by flow cytometry. Values were expressed relative to the fluorescence signal of the control.

\section{Mitochondrial function analysis}

Cells from each experimental condition were stained with tetramethylrhodamine ethyl ester (TMRE) reagent, MitoSOX Red and MitoTracker Green (Molecular Probes) at $37^{\circ} \mathrm{C}$ for 30 min for detection of the mitochondrial membrane potential, mitochondrial ROS and mitochondrial mass, respectively. Cells were washed twice with PBS, trypsinized and collected, 
and the pellet was re-suspended in PBS for analysis using flow cytometry.

\section{Oxygen uptake measurements}

Mitochondrial oxygen consumption was measured in a Seahorse XF24 Extracellular Flux analyzer (Seahorse Bioscience, MA, USA) after calibration by air saturation. The electrode was calibrated between 0 and $100 \%$ saturation with atmospheric oxygen at $37^{\circ} \mathrm{C}$. Mitochondrial complex I, III and $\mathrm{V}$ inhibitors (rotenone $(2 \mu \mathrm{M})$, oligomycin $(0.5 \mu \mathrm{M})$ and carbonyl cyanide-4-(trifluoromethoxy)phenylhydrazone (FCCP) $(2 \mu \mathrm{M})$, respectively) were injected into the wells, followed by the measurement cycles for the oxygen consumption rate (OCR). The OCR was normalized to the cell number.

\section{Immunofluorescence labeling and immunoblotting assay}

The immunoassay was performed as previously described (Li et al. 2015a).

\section{Cell death detection by Annexin V-FITC/PI double staining}

Analysis of the cell death of each condition was performed using an Alexa Fluor Annexin V/Dead Cell Apoptosis kit (Molecular Probes) according to the manufacturer's protocol. Briefly, cells were harvested after treatment. Cells were then washed twice with cold binding buffer, re-suspended in binding buffer and stained with $5 \mu \mathrm{L}$ each of Annexin V-FITC and PI solution for $15 \mathrm{~min}$ at room temperature in the dark. After incubation, $1 \mathrm{~mL}$ binding buffer was added, and cells were analyzed by flow cytometry (FACSCalibur, BD Bioscience).

\section{Antibodies and inhibitors}

Anti-PARP (100573), anti-BAX (109683), anti-BCL2 (100064), anti-cytochrome $C$ (108585), anti- $\alpha$-tubulin (112141), antiRIP-1 (111074) and anti-RIP-3 (107574) were purchased from GeneTex. (ICON-Genetex Inc., Taipei, Taiwan); Anti-caspase-9 (ab115161), anti-caspase-3 (ab90437), Necrostatin-1 (ab141053) and z-VAD-FMK (ab120382) were purchased from Abcam. Anti- $\beta$-actin (A5411) was purchased from Sigma.

\section{Statistical analysis}

Each experiment was performed at least 6 times, and all data are represented as means \pm standard deviation (s.D.) of quadruplicate measurements. The intensity of bands in Western blots or fluorescence were quantified using AlphaDigiDoc software (Cell Biosciences, ON, Canada), ImageJ software (NIH) and MicroP software (Peng et al. 2011). The intensity value for each protein was normalized against the intensity of the loading control for that same sample. The values after normalizing to loading control in the control groups were set as 1.0. Statistical significance was evaluated using two-way analysis of variance test using GraphPad Prism, version 6.0 (GraphPad Software), followed by Duncan's multiple range test (MRT), to assess the differences between group means. Differences were considered significant when $P<0.05$.

\section{Results}

\section{DHEA protected HO23 cells against serum deprivation-induced cell death}

Initially, we evaluated whether DHEA could inhibit apoptosis of HO23 cells. We used a serum-free (SF) medium as an apoptosis inducer (Goyeneche et al. 2006). To test whether DHEA was able to protect cells from serum deprivation-induced apoptosis, $\mathrm{HO} 23$ cells were incubated in SF medium for $24 \mathrm{~h}$ with or without DHEA. In the DHEA-treated group, we treated cells with various concentrations of DHEA at the last $20 \mathrm{~h}$ in SF medium (Fig. 1A). As shown in Fig. 1B, administration of DHEA enhanced cell viability during serum deprivation. The effect of DHEA was concentration dependent, with DHEA concentrations of $1 \mathrm{nM}$ and $10 \mathrm{nM}$ inducing the maximal protective effect. DHEA concentration of $1 \mathrm{nM}$ was used for further studies. As shown in Fig. 1C, serum
A
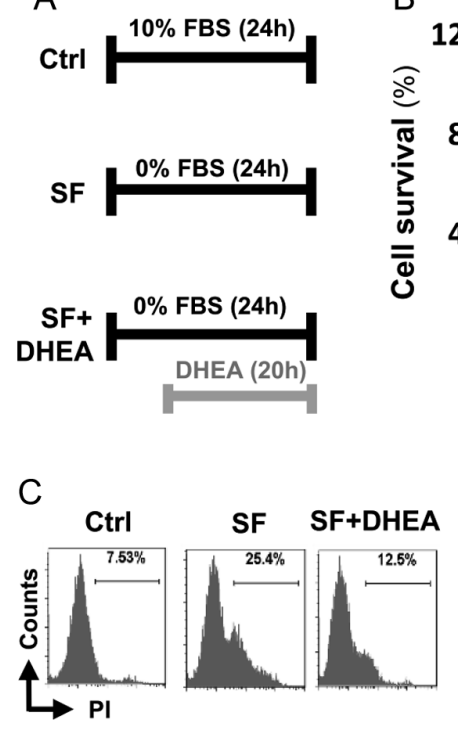

$\mathrm{B}$
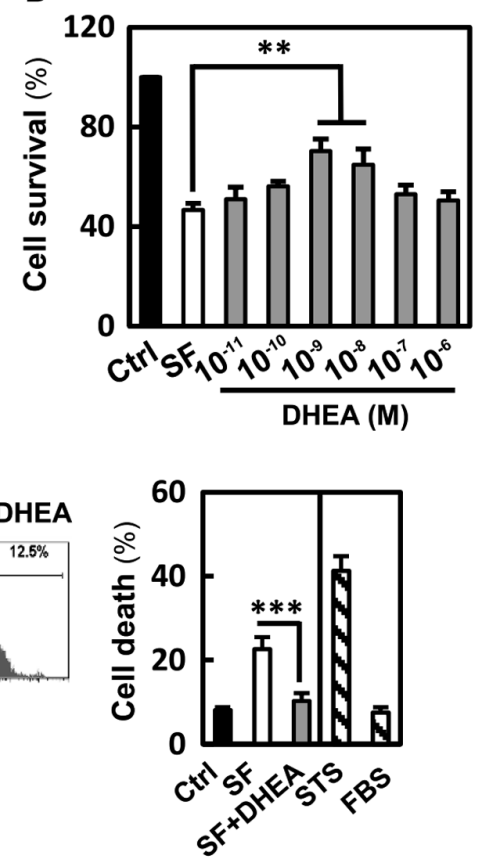

Figure 1 Effect of DHEA inhibited starvation-induced proliferation inhibition in vitro. (A) Flow chart for in vitro experimental design and treatment schedule. (B) HO23 cells were treated with different concentrations of DHEA against serum deprivation-induced decreased proliferation. (C) HO23 cells were treated with DHEA for $20 \mathrm{~h}$ and subsequently analyzed using flow cytometry with propidium iodide $(\mathrm{PI})$ staining for live cells to determine the populations with cell death. Quantitative data were evaluated by the PI-positive population of individual histograms. Cells cultured in a medium with $5 \%$ FBS and staurosporine (STS) served as the positive and negative control, respectively. ${ }^{* *} P<0.01$ and ${ }^{* *} P<0.001$ compared with the serum-free (SF) group. 

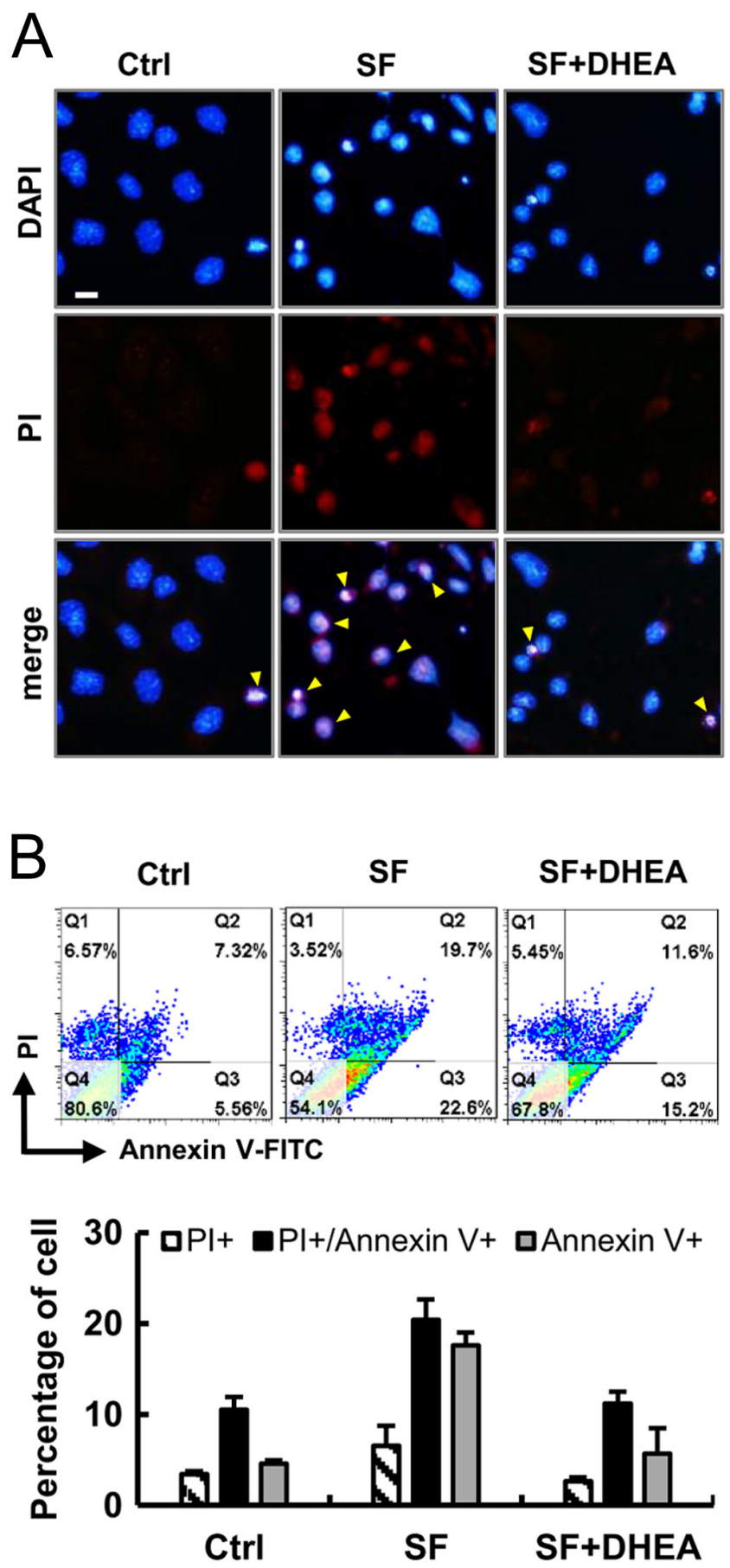

Figure 2 DHEA protects granulosa cells against apoptosis and necrosis. (A) Cells were stained with Hoechst 33342 (blue) and PI (red) for live-cell imaging and monitored. Yellow arrowheads indicate apoptotic and necrotic-like cells. (B) Annexin V/PI double staining was used to detect the PS out-flipping phenomenon, one of the clearest characteristics of apoptotic and necrotic cell death by flow cytometry.

deprivation remarkably increased the percentage of $\mathrm{Pl}$-positive cells by live-cell fluorescent $(7.53 \%$ in serum vs $25.4 \%$ in the SF condition). This effect was effectively reversed by DHEA, which reduced the percentage of Pl-stained cells to control levels.

\section{Treatment of DHEA protects $\mathrm{HO} 23$ cells against apoptosis and necrosis}

The nuclear morphology of $\mathrm{HO} 23$ cells after various treatments was observed to stain with Hoechst dye and PI double staining for live-cell imaging. No abnormal cell was found in the control group while a high number of cells displayed typical apoptotic (nuclear shrinkage, apoptotic bleb and irregular shape) and necrosislike (cell swelling, plasma membrane rupture and detachment) morphological changes in SF treated cells (Fig. 2A). However, DHEA treatment thus suppressed the starvation-induced apoptosis and necrosis of GCs (Fig. 2A). In Fig. 2B, the Annexin V/PI-positive cells were counted as the apoptotic cells. Our results showed that the percentage of apoptotic and necrotic cells in starvation group was dramatically increased compared with that in the control. And treatment with DHEA markedly suppressed the starvation-induced apoptosis and necrosis (Fig. 2B).

\section{DHEA ameliorates a starvation-induced mitochondrial dysfunction}

To explore the protective effect of DHEA on mitochondrial functions, $\mathrm{HO} 23$ cells were stained with fluorescent probe DCFDA and MitoSOX, which detect hydrogen peroxide and mitochondrial ROS, respectively. Cells in the starvation group showed higher green and red fluorescence induced by starvation compared with that in the normal control group. A flow cytometry analysis was performed to quantify the effect of DHEA reduced intracellular and mitochondrial ROS generation, and the results are presented in Fig. 3A and B. The difference between the control and the starvation group was significant $(P<0.01)$; however, treatment of DHEA, the ROS level was effectively decreased and both intracellular and mitochondrial ROS nearly down to normal value. From this finding, it appears that the DHEA protects the HO23 cells from oxidative stress injury, by inhibiting the mitochondrial ROS generation. We further characterized its effect on the mitochondrial membrane potential $(\Delta \Psi m)$ and mitochondrial mass. As shown in Fig. 3C, serum deprivation resulted in a dramatic reduction of fluorescence, indicating a loss of $\Delta \Psi m$ and mitochondrial damage. We investigated whether DHEA protection of mitochondrial mass is related to the duration of starvation. We observed that cells treated with DHEA had $102.2 \%$ protection in maintaining the mitochondrial mass, after starvation incubated (Fig. 3D). These results suggested that DHEA reduced mitochondrial ROS and improves their mass and function. 
A
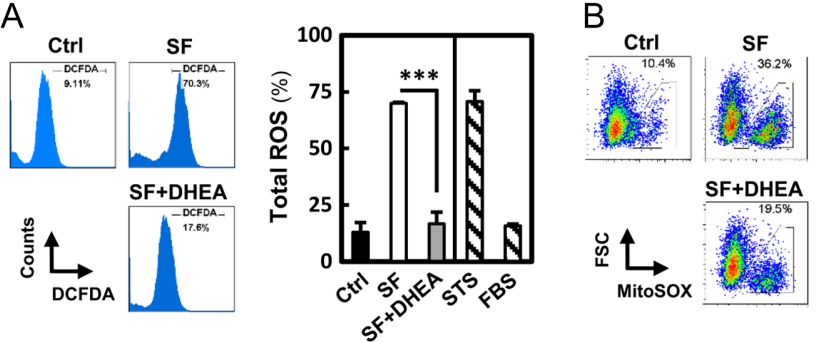

C

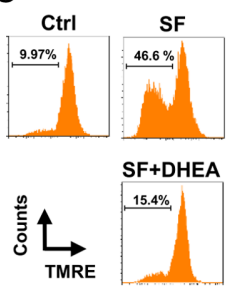

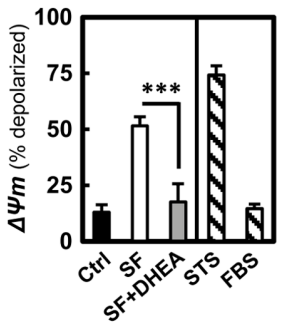

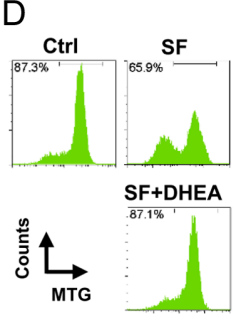

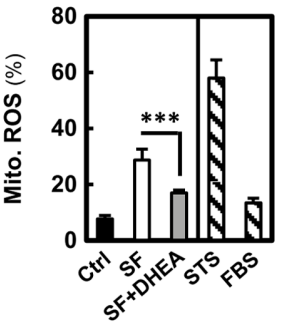

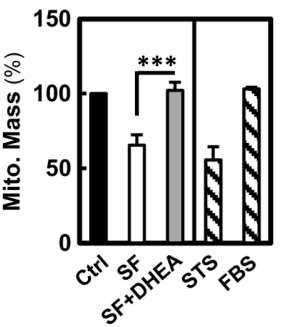

Figure 3 DHEA ameliorated mitochondrial function and helped maintaining mitochondrial mass. Flow cytometry analysis for (A) intracellular hydrogen peroxide (B) mitochondrial reactive oxidative species (ROS), (C) mitochondrial membrane potential $(\triangle \Psi m)$ and $(\mathrm{D})$ mitochondrial mass in $\mathrm{HO} 23$ cells among control, serum-free (SF) and DHEA-treated groups. Quantitative analysis of the ROS, mitochondrial membrane potential and mitochondrial mass in $\mathrm{HO} 23$ cells was calculated. Cells cultured in a medium with $5 \% \mathrm{FBS}$ and staurosporine (STS) served as the positive and negative control, respectively. *** $P<0.001$ compared with the SF group.

\section{DHEA enhanced mitochondrial function in $\mathrm{HO} 23$ cells}

To evaluate the effect of mitochondrial function, cellular bioenergetics was measured in cells after DHEA treatment. We observed that cells treated with DHEA maintained their mitochondrial mass and increased their mitochondrial DNA (Figs 3D and 4A). We further examined the effects of DHEA treatment on mitochondrial respiration via real-time measurement of the OCR. As shown in Fig. 4B and C, decreased mitochondrial respiration efficiency was observed in the SF group as evident by decreased basal respiration and maximal respiratory capacity. The OCR of the basal and maximal respiration significantly increased in the DHEA treatment group. These results suggest that DHEA enhances mitochondrial biogenesis and helps maintain their mass.

\section{DHEA suppressed serum deprivation-induced apoptosis in $\mathrm{HO} 23$ cells}

To explore the mechanisms of reducing apoptosis following DHEA treatment in $\mathrm{HO} 23$ cells, activation of caspases and expression of pro-apoptotic proteins were analyzed using Q-PCR, Western blot and immunoassay. DHEA treatment significantly decreased the expression of BAX, BCL2, caspase-9, caspase-3, and cytochrome C RNA and protein levels (Fig. 5A and B). In addition, immunostaining was used to confirm the cellular distribution of cytochrome c. Cell mitochondria were primarily identified using MitoTracker red and were later labeled with FITC-conjugated cytochrome $c$ antibodies (Fig. 5C). DHEA reduced cytochrome $c$ release from mitochondria to the cytosol. As shown in Fig. 5D, addition of DHEA resulted in a significant increase of overlap
A
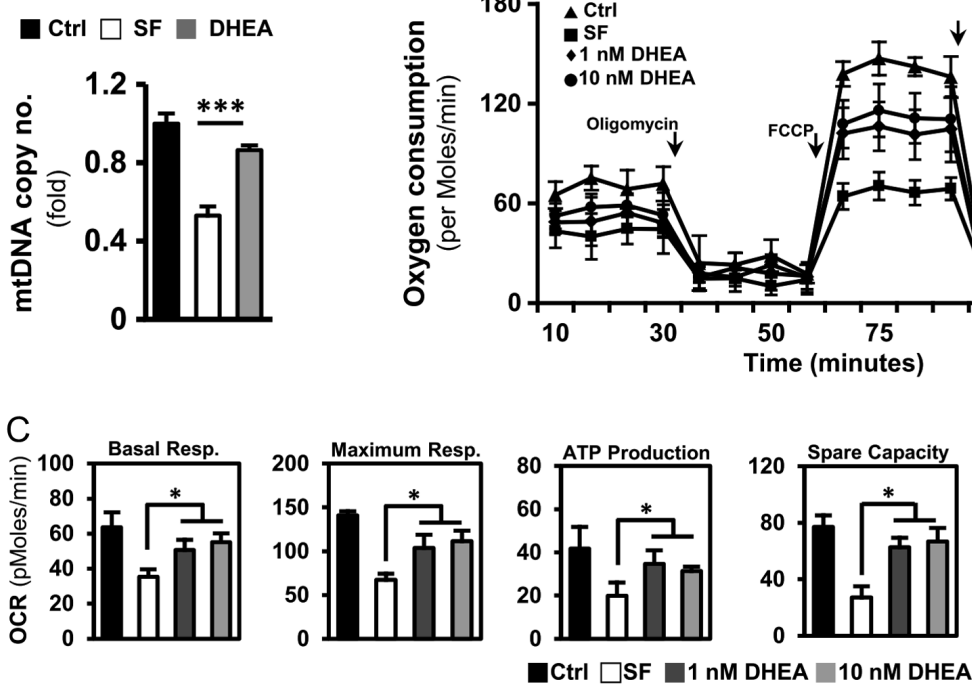

Figure 4 DHEA increased cellular oxygen consumption in vitro. (A) Q-PCR analysis for mitochondrial DNA (mtDNA) copy number, as shown by the fold increase above the control. (B) Mitochondrial function was determined by OCR utilizing the Seahorse Bioscience Analyzer. (C) All OCR values at different phases of basal respiration, maximal respiration, ATP-coupled respiration and spare capacity were recorded, and the values were calculated among the control, SF and DHEA-treated groups. DHEA treatment promoted OCR and increased mitochondrial respiration compared with the SF condition. Cells cultured in a medium with 5\% FBS and staurosporine (STS) served as the positive and negative control, respectively. ${ }^{*} P<0.05$ and $* * * P<0.001$ compared with the SF group. 


\section{A $\square$ Ctrl $\square$ SF $\square$ SF+DHEA}
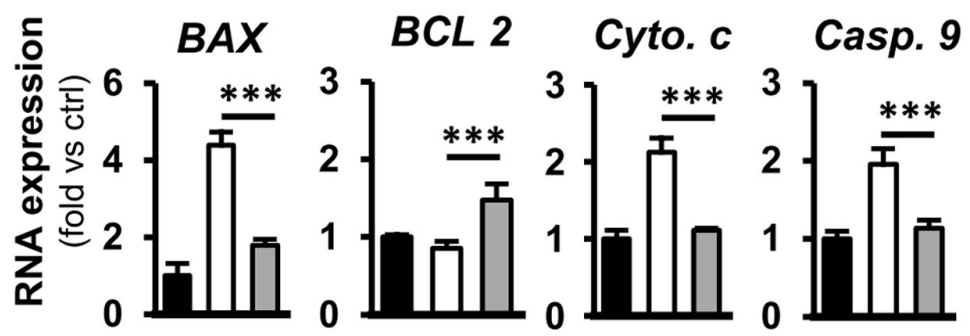

Casp. 3

C
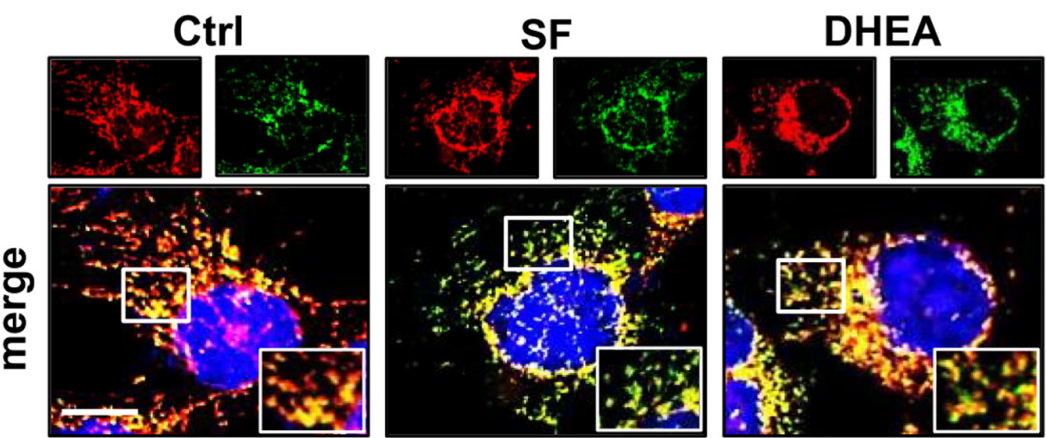

MitoTracker / Cyto. c / DAPI

\section{$\mathrm{D}$}
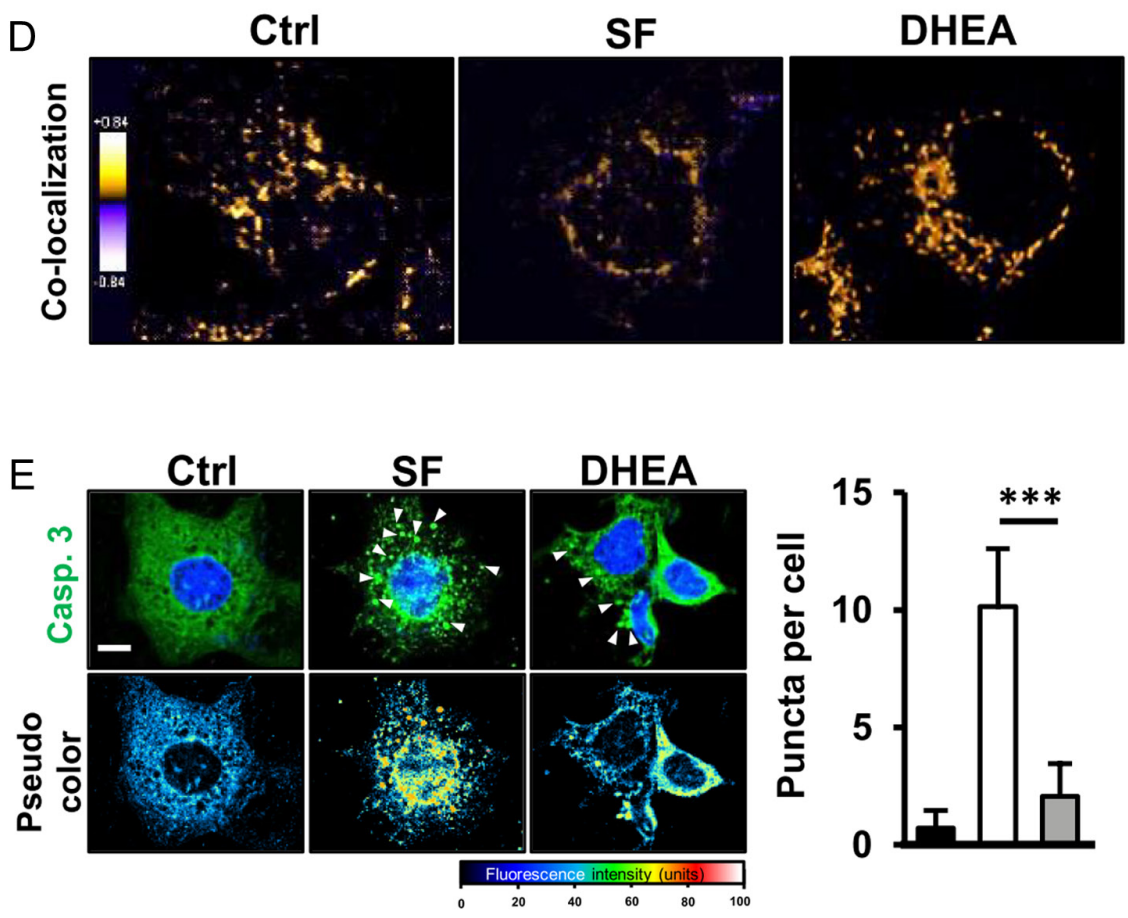

$\mathrm{F}$

Figure 5 DHEA protected HO23 against starvation-induced intrinsic apoptosis. (A) Real-time PCR analysis for mRNA expression of apoptotic relative genes (BAX, BCL2, cytochrome $c$, caspase-3 and caspase-9) in HO23 cells. (B) Western blot analysis of relative apoptotic protein in HO23 cells. Protein expression levels were normalized to beta-actin and expressed as the fold change to the respective control.

(C) Representative immunofluorescence of cytochrome c (green) and MitoTracker (red) using a FITC-conjugate (green) antibody. (D) Calculation and quantification of Pearson's co-localization coefficients between MitoTracker red and cytochrome $c$ for each group. The coefficients were generated using ImageJ software. (E) Immunostaining of caspase 3 using a FITC-conjugated antibody (green) in DHEA-treated HO23 cells. Caspase-3 was quantified by counting FITC fluorescent puncta per cell. The results are the means of three independent experiments. Twenty cells were analyzed per assay. (F) The survival of HO23 cells with DHEA treatment at the presence or absence of z-VAD following starvation treatment. Scare bar $=20 \mu \mathrm{m} .{ }^{* *} P<0.01$ and ${ }^{* * * P}<0.001$ compared with the SF group. 

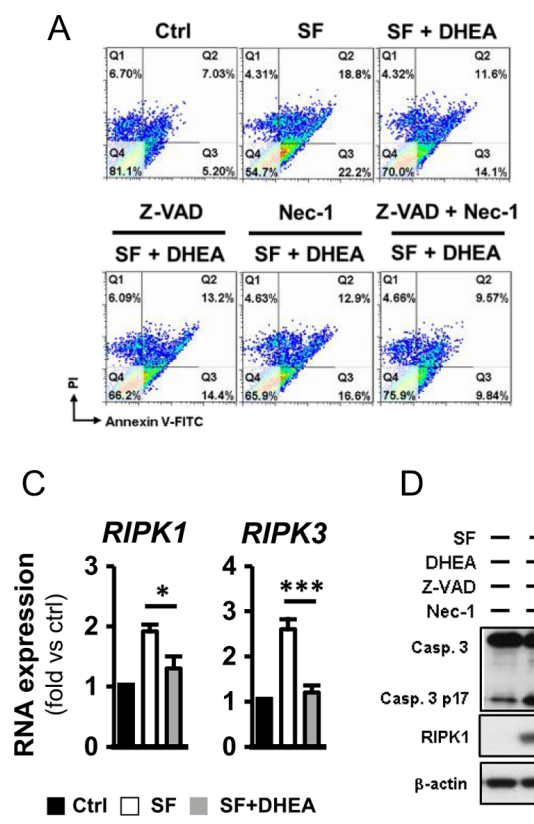

D
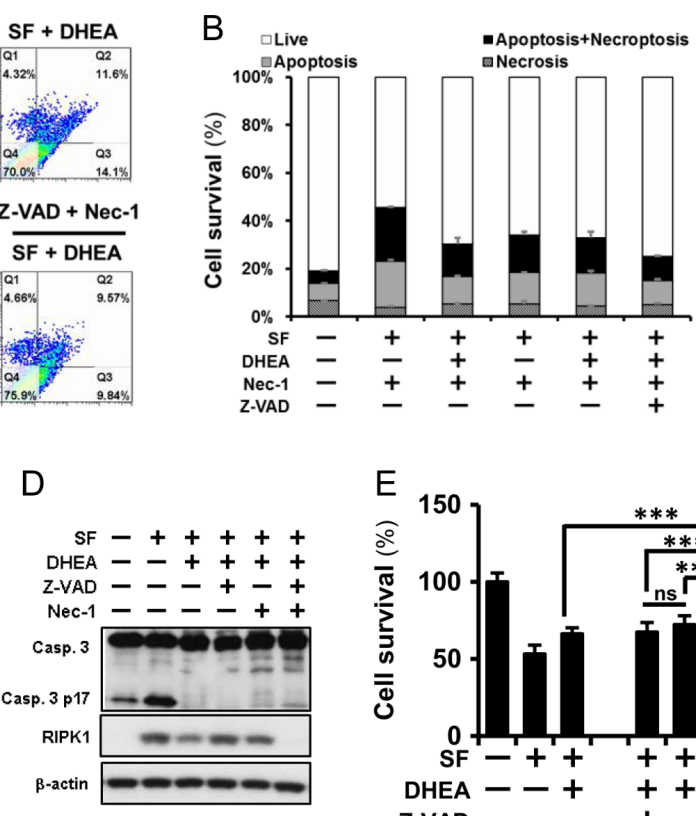

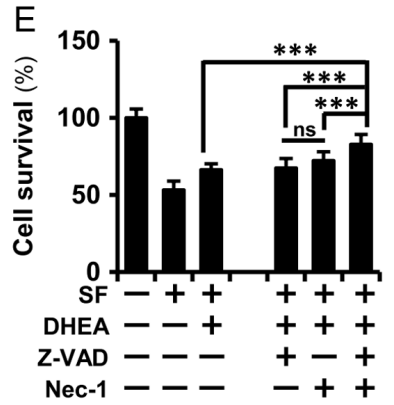

Figure 6 DHEA attenuated necroptosis in the presence of pan-caspase and RIPK-1 inhibitor. (A \& B) HO23 cells were treated with DHEA in the presence or absence of necrostatin 1 and z-VAD for 120 min during starvation. Dot plot distribution of cells stained with Annexin V-FITC and PI analyzed by flow cytometry. The values and quantification indicate the percentage of cells in each region. (C) Real-time PCR analysis for mRNA expression of apoptotic relative genes (RIPK1 and 3 ) in HO23 cells. (D) Western blot analysis of RIPK1 and caspase-3 in HO23 cells. Protein expression levels were normalized to

beta-actin and expressed as the fold change to the respective control. (E) CCK-8 value of proliferation $\mathrm{HO} 23$ cells with DHEA administration in the presence or absence of Necrostatin 1 and z-VAD during starvation. $* P<0.05$ and ${ }^{* * *} P<0.001$. coefficient between mitochondria and cytochrome $c_{\text {, }}$ while DHEA treatment reduced SF-induced cytochrome $C$ release to the cytosol. We also confirmed the cellular distribution of caspase 3. In DHEA-treated cells, we observed a decrease in the number of puncta per cell (Fig. 5E). Moreover, there was no change in cell viability after treatment with $50 \mu \mathrm{M}$ of the pan-caspase inhibitor (z-VAD), indicating that caspase may be the factor involved in SF-induced cell death (Fig. 5F). These results verified the protective effect of DHEA treatment on preventing mitochondrial dysfunction associated with the intrinsic apoptotic pathway.

\section{DHEA protects human granulosa cells against necroptosis}

To further explore whether the protective mechanisms of DHEA suppresses necroptosis and apoptosis, we tested the effect of the RIP1 inhibitor (Nec-1) and caspase inhibitor (z-VAD) by staining with Annexin V/ PI by flow cytometry. In previous reports, dual staining with Annexin V/PI was used to assess apoptotic cell death, in which Annexin $\mathrm{V}^{+} / \mathrm{Pl}^{-}$cells were regarded as early apoptotic cells, and double-positive cells were determined as late apoptotic or necroptic cells (Vermes et al. 1995). Apoptosis and necrosis are mediated by distinct but overlapping pathways involving cell surface death receptors and cellular characteristic (Nikoletopoulou et al. 2013). As Fig. 6A shows, the different types of cell death were classified according to Annexin $\mathrm{V}^{+}$as apoptosis, $\mathrm{Pl}^{+}$as necrosis and double positive as apoptosis and necroptosis. Dual staining demonstrated that DHEA protected necroptotic cell death was almost completely inhibited by Nec1 and partially hampered the number of double-positive region cells further increased $\mathrm{PI}^{+}$cells. Pretreatment with z-VAD also completely suppressed the appearance of $\mathrm{Pl}^{+}$cells as well as double-positive cells in SF group, while DHEA decreased Annexin $\mathrm{V}^{+}$region cells. Accordingly, we suggest that DHEA prevented starvation-mediated not only apoptotic but also necroptotic cells show presence or absence of Nec1 or/and z-VAD. Nec1 or/and z-VAD treatment might promote the survival of HO23 cells dying under necroptotic and apoptosis conditions. To further explore the mechanisms of protective effects on necroptosis, we analyzed the effect of the DHEA under starvation conditions by Q-PCR and Western blotting. The results showed that SF treatment significantly increased the expression of RIPK1 and RIPK3, compared with the control (Fig. 6B and C). While DHEA markedly attenuated the SF-induced upregulation of RIP kinase. The results of necrosome complex were further supported by flow cytometry (Fig. 6A). Taken together, the findings suggest that DHEA can inhibit the activation of RIP kinase in SF-induced HO23 cells through the necroptosis pathway. Interestingly, Nec1 plus z-VAD almost completely hampered the SF-induced necroptotic and apoptotic cell death measured by Cell Counting Kits-8. In Fig. 6D, we show that treatment of DHEA significantly increased cell viability compared with the SF group. The combination of Nec1 and z-VAD slightly increased cell survival from $66.8 \%$ (DHEA group) to $82.8 \%$ (Nec1 + z-VAD + DHEA group).

\section{Discussion}

The ovary is a complex endocrine gland responsible for production of sex steroids and is the source of 
fertilizable ova for reproduction. Understanding the mechanisms that regulate ovarian steroidogenesis and folliculogenesis at the molecular and cellular level requires readily available cells for in vitro studies. For humans, the major source of GCs for in vitro studies has been patients undergoing in vitro fertilization (IVF). The development of granulosa cell lines has provided a readily accessible, reproducible and renewable model for studying the molecular events regulating granulosa cell steroidogenesis and folliculogenesis (Havelock et al. 2004).

The results of the current study showed the protective effect and improve mitochondrial functions of DHEA on human ovary granulosa $\mathrm{HO} 23$ cells during starvation. In the present study, we hypothesized that administration of DHEA could protect an effect on starvation-induced oocyte granulosa cell death. To test our hypothesis, we simulated starvation in human granulosa cell line $\mathrm{HO} 23$, and then, we examined the potential effects of DHEA on serum deprivation-induced cell death. In addition, we also explored the potential mechanism of DHEA on cell death in starvation model. In our study, we found that cell death of $\mathrm{HO} 23$ cells, determined by Hoechst staining and flow cytometry with Annexin $\mathrm{V}$ and $\mathrm{PI}$ staining (Fig. 2A and B), was significantly increased during starvation. However, we found cell proliferation was markedly improved in starvation of HO23 cells after using pan-caspase and necroptosis inhibitor (Fig. 5). These results suggest that both z-VAD-FMK and necrostatin- 1 have protective effect on $\mathrm{HO} 23$ cells. We were able to demonstrate that mitochondrial mass in DHEA-treated cells were nearly able to recover as compared with control group (Fig. 3D). In the molecular mechanism, we found that starvation-triggered cellular and mitochondrial ROS resulted in BAX activation, as indicated by a significantly increased association with mitochondria (Fig. 3B). Therefore, the inhibition of BAX mitochondrial translocation by DHEA may occur primarily through changes in cytosolic ROS.

In order to elucidate whether the signaling pathways in DHEA prevent mitochondria against cell death, we clarified two major pathways: caspasedependent pathway (Fig. 4) and programmed necrosis pathway (Fig. 5). Caspase-dependent pathway has been determined as part of the signaling pathways in apoptosis by starvation. Serum deprivation compromised mitochondrial function as evidenced by the release of cytochrome $C$ and AIF, BAX and Drp-1 translocation to mitochondria and the loss of mitochondrial membrane potential (Schamberger et al. 2005). Necroptosis is a type of programmed cell death (PCD) that has features of both apoptosis and necrosis (Vandenabeele et al. 2010). Mitochondrial damage causes apoptosis and necroptosis through the caspasedependent or -independent pathway (Piwocka et al. 2001, Baritaud et al. 2010, Seshadri et al. 2011). In contrast, excessive ROS suppresses cleavage of caspases and mediates cell death through necroptosis pathway (Wu et al. 2012). Despite the reports presented, the relationship between mitochondrial damage and necrosome formation during necroptosis, its mechanism has been largely unknown and particularly the crosstalk of apoptosis and necroptosis on GCs during starvation.

The protection effect of DHEA was also demonstrated in preventing mitochondrial dysfunction during starvation. Together, our data indicate that the DHEA treatment is a potential anti-cell death agent and merits further investigation. At least two activities of DHEA may contribute to its mitochondrial protective effects in human GCs. Firstly, DHEA not only directly suppresses the DCFHDA and MitoSOX (Fig. 3A and B) but also increases proliferation and mitochondrial mass on GCs (Figs 1B and 4D). Secondly, mitochondrial dysfunction induces opening of the mitochondrial permeability transition pores (PTP), dissipation of $\Delta \Psi m$ and release of apoptogenic proteins such as cytochrome $c$, AIF and endonuclease G (Tait \& Green 2010). We have found that DHEA could inhibit the depolarization of mitochondrial $\Delta \Psi m$ (Fig. 3C). It is known that $\Delta \Psi m$ plays diverse roles in cell physiology and pathology including regulation of apoptosis and necroptosis (Richter 1998, Vanden Berghe et al. 2010). The inhibition of mitochondrial $\Delta \Psi m$ depolarization in human GCs can lead to the suppression of apoptotic relative factors release. We found that DHEA reduced the disruption of mitochondrial functions and induced apoptosis in $\mathrm{HO} 23$ cells. More specifically, DHEA prevented the release of cytochrome $c$ from the mitochondria into the cytosol (Fig. 4C), which led to the formation of conducting channels in the outer mitochondrial membrane (Ott et al. 2009).

Previous studies have shown that not only DHEA, but also many hormones, inhibit apoptosis, and they are considered to act through steroid receptors (Gu et al. 2001, Sudoh et al. 2001). However, the action of DHEA may be partially mediated by its metabolites because HO23 GCs could express several metabolic enzymes, such as $3 \beta \mathrm{HSD}$ and HSD17 $\beta 3$, which were involved in DHEA metabolism. However, the detailed molecular mechanism and signaling pathways of these effects of DHEA have not yet been elucidated. While DHEA is enzymatically metabolized to androgens and estrogens, it is not clear whether DHEA exerts its effects directly to PCD. With regard to the direct inhibitory effect on cells, our data show that DHEA could inactivate the RIPK-1 and -3 during starvation. It has been shown that key molecular mediators including RIP kinase promote necroptosis by activating multiple signal pathways, such as the PARP-1/RIPK/MLKL pathway for directly regulating GCs. Furthermore, DHEA pretreatment significantly reduced the expression of BAX/caspase in SF-induced HO23 cells and neutralized the cell death. Our data showed that DHEA decreases both apoptosis and necroptosis without the induction of mitochondrial ROS generation, indicating that DHEA may directly 
and indirectly regulate the multiple signal transduction pathways through mitochondrial damage in the GCs.

Our data showed that DHEA decreases both apoptosis and necroptosis without the induction of mitochondrial ROS generation, indicating that DHEA may directly and indirectly regulate the multiple signal transduction pathways through mitochondrial damage in the GCs.

\section{Conclusions}

To our knowledge, this is the first study to show that DHEA can ameliorate the mitochondrial triggered cell death of HO23 cells by inducing caspase-dependent apoptosis and necroptosis.

\section{Declaration of interest}

The authors declare that there is no conflict of interest that could be perceived as prejudicing the impartiality of the research reported.

\section{Funding}

This research did not receive any specific grant from any funding agency in the public, commercial or not-for-profit sector.

\section{Acknowledgments}

This work was generously supported by grants VGHKS105-G06-01 from Kaohsiung Veterans General Hospital and MOST 103-2314-B-010-043-MY3 from the Ministry of Science and Technology, Executive Yuan.

\section{References}

Anderson RA, Sciorio R, Kinnell H, Bayne RA, Thong KJ, de Sousa PA \& Pickering S 2009 Cumulus gene expression as a predictor of human oocyte fertilisation, embryo development and competence to establish a pregnancy. Reproduction 138 629-637. (doi:10.1530/REP-09-0144)

Baritaud M, Boujrad H, Lorenzo HK, Krantic S \& Susin SA 2010 Histone H2AX: the missing link in AIF-mediated caspase-independent programmed necrosis. Cell Cycle 9 3166-3173. (doi:10.4161/ cc.9.16.12887)

Blohberger J, Kunz L, Einwang D, Berg U, Berg D, Ojeda SR, Dissen GA, Frohlich T, Arnold GJ, Soreq H et al. 2015 Readthrough acetylcholinesterase (AChE-R) and regulated necrosis: pharmacological targets for the regulation of ovarian functions? Cell Death and Disease 6 e1685. (doi:10.1038/cddis.2015.51)

Cillo F, Brevini TA, Antonini S, Paffoni A, Ragni G \& Gandolfi F 2007 Association between human oocyte developmental competence and expression levels of some cumulus genes. Reproduction 134 645-650. (doi:10.1530/REP-07-0182)

Corn CM, Hauser-Kronberger C, Moser M, Tews G \& Ebner T 2005 Predictive value of cumulus cell apoptosis with regard to blastocyst development of corresponding gametes. Fertility and Sterility $\mathbf{8 4}$ 627-633. (doi:10.1016/j.fertnstert.2005.03.061)

Fragouli E, Lalioti MD \& Wells D 2014 The transcriptome of follicular cells: biological insights and clinical implications for the treatment of infertility. Human Reproduction Update 20 1-11. (doi:10.1093/ humupd/dmt044)

Goyeneche AA, Harmon JM \& Telleria CM 2006 Cell death induced by serum deprivation in luteal cells involves the intrinsic pathway of apoptosis. Reproduction 131 103-111. (doi:10.1530/rep.1.00751)

Gu Y, Zou Y, Aikawa R, Hayashi D, Kudoh S, Yamauchi T, Uozumi H, Zhu W, Kadowaki T, Yazaki Y et al. 2001 Growth hormone signalling and apoptosis in neonatal rat cardiomyocytes. Molecular and Cellular Biochemistry 223 35-46. (doi:10.1023/A:1017941625858)

Havelock JC, Rainey WE \& Carr BR 2004 Ovarian granulosa cell lines. Molecular and Cellular Endocrinology 228 67-78. (doi:10.1016/j. mce.2004.04.018)

Hosokawa K, Dantes A, Schere-Levy C, Barash A, Yoshida Y, Kotsuji F, Vlodavsky I \& Amsterdam A 1998 Induction of Ad4BP/ SF-1, steroidogenic acute regulatory protein, and cytochrome P450scc enzyme system expression in newly established human granulosa cell lines. Endocrinology 139 4679-4687. (doi:10.1210/ endo.139.11.6279)

Host E, Gabrielsen A, Lindenberg S \& Smidt-Jensen S 2002 Apoptosis in human cumulus cells in relation to zona pellucida thickness variation, maturation stage, and cleavage of the corresponding oocyte after intracytoplasmic sperm injection. Fertility and Sterility 77 511-515. (doi:10.1016/S0015-0282(01)03006-0)

Hussein MR 2005 Apoptosis in the ovary: molecular mechanisms. Human Reproduction Update 11 162-177. (doi:10.1093/humupd/dmi001)

Labrie F 2015 All sex steroids are made intracellularly in peripheral tissues by the mechanisms of intracrinology after menopause. Journal of Steroid Biochemistry and Molecular Biology 145 133-138. (doi:10.1016/j. jsbmb.2014.06.001)

Lan KC, Chang SY, Huang FJ, Lin HJ, Lin CY, Huang KE \& Kang HY 2013 Analysis of androgen receptor and anti-Mullerian hormone pathways in human granulosa cells under luteinizing hormone treatment. Reproductive Biology and Endocrinology 11 11. (doi:10.1186/14777827-11-11)

Lee KS, Joo BS, Na YJ, Yoon MS, Choi OH \& Kim WW 2001 Cumulus cells apoptosis as an indicator to predict the quality of oocytes and the outcome of IVF-ET. Journal of Assisted Reproduction and Genetics 18 490-498. (doi:10.1023/A:1016649026353)

Lee SK, Zhao MH, Kwon JW, Li YH, Lin ZL, Jin YX, Kim NH \& Cui XS 2014 The association of mitochondrial potential and copy number with pig oocyte maturation and developmental potential. Journal of Reproduction and Development 60 128-135. (doi:10.1262/jrd.2013-098)

Li CJ, Sun LY \& Pang CY 2015a Synergistic protection of N-acetylcysteine and ascorbic acid 2-phosphate on human mesenchymal stem cells against mitoptosis, necroptosis and apoptosis. Scientific Reports 59819. (doi:10.1038/srep09819)

Li SH, Lin MH, Hwu YM, Lu CH, Yeh LY, Chen YJ \& Lee RK 2015b Correlation of cumulus gene expression of GJA1, PRSS35, PTX3, and SERPINE2 with oocyte maturation, fertilization, and embryo development. Reproductive Biology and Endocrinology 13 93. (doi:10.1186/s12958-015-0091-3)

Lin LT, Tsui KH \& Wang PH 2015 Clinical application of dehydroepiandrosterone in reproduction: a review of the evidence. Journal of the Chinese Medical Association 78 446-453. (doi:10.1016/j. jcma.2014.12.008)

Linkermann A, Brasen JH, Himmerkus N, Liu S, Huber TB, Kunzendorf U \& Krautwald S 2012 Rip1 (receptor-interacting protein kinase 1) mediates necroptosis and contributes to renal ischemia/reperfusion injury. Kidney International 81 751-761. (doi:10.1038/ki.2011.450)

Nagels HE, Rishworth JR, Siristatidis CS \& Kroon B 2015 Androgens (dehydroepiandrosterone or testosterone) for women undergoing assisted reproduction. Cochrane Database of Systematic Reviews 11 Cd009749. (doi:10.1002/14651858.CD009749.pub2)

Nikoletopoulou V, Markaki M, Palikaras K \& Tavernarakis N 2013 Crosstalk between apoptosis, necrosis and autophagy. Biochimica et Biophysica Acta 1833 3448-3459. (doi:10.1016/j.bbamcr.2013.06.001)

Ott M, Norberg E, Zhivotovsky B \& Orrenius S 2009 Mitochondrial targeting of tBid/Bax: a role for the TOM complex? Cell Death and Differentiation 16 1075-1082. (doi:10.1038/cdd.2009.61)

Peng JY, Lin CC, Chen YJ, Kao LS, Liu YC, Chou CC, Huang YH, Chang FR, Wu YC, Tsai YS et al. 2011 Automatic morphological subtyping reveals new roles of caspases in mitochondrial dynamics. PLoS Computational Biology 7 e1002212. (doi:10.1371/journal.pcbi.1002212) 
Piwocka K, Jaruga E, Skierski J, Gradzka I \& Sikora E 2001 Effect of glutathione depletion on caspase-3 independent apoptosis pathway induced by curcumin in Jurkat cells. Free Radical Biology and Medicine 31 670-678. (doi:10.1016/S0891-5849(01)00629-3)

Ramalho-Santos J, Varum S, Amaral S, Mota PC, Sousa AP \& Amaral A 2009 Mitochondrial functionality in reproduction: from gonads and gametes to embryos and embryonic stem cells. Human Reproduction Update 15 553-572. (doi:10.1093/humupd/dmp016)

Richter C 1998 Oxidative stress, mitochondria, and apoptosis. Restorative Neurology and Neuroscience 12 59-62. (doi:10.1007/s00204-0131034-4)

Roy MJ, Vom A, Czabotar PE \& Lessene G 2014 Cell death and the mitochondria: therapeutic targeting of the BCL-2 family-driven pathway. British Journal of Pharmacology 171 1973-1987. (doi:10.1111/ bph.12431)

Santos TA, El Shourbagy S \& St John JC 2006 Mitochondrial content reflects oocyte variability and fertilization outcome. Fertility and Sterility 85 584-591. (doi:10.1016/j.fertnstert.2005.09.017)

Schamberger CJ, Gerner C \& Cerni C 2005 Caspase-9 plays a marginal role in serum starvation-induced apoptosis. Experimental Cell Research 302 115-128. (doi:10.1016/j.yexcr.2004.08.026)

Seshadri P, Rajaram A \& Rajaram R 2011 Plumbagin and juglone induce caspase-3-dependent apoptosis involving the mitochondria through ROS generation in human peripheral blood lymphocytes. Free Radical Biology and Medicine 51 2090-2107. (doi:10.1016/j. freeradbiomed.2011.09.009)

Smith CC, Davidson SM, Lim SY, Simpkin JC, Hothersall JS \& Yellon DM 2007 Necrostatin: a potentially novel cardioprotective agent? Cardiovascular Drugs and Therapy 21 227-233. (doi:10.1007/s10557007-6035-1)

Sudoh N, Toba K, Akishita M, Ako J, Hashimoto M, lijima K, Kim S, Liang YQ, Ohike Y, Watanabe T et al. 2001 Estrogen prevents oxidative stressinduced endothelial cell apoptosis in rats. Circulation 103 724-729. (doi:10.1161/01.CIR.103.5.724)

Tait SW \& Green DR 2010 Mitochondria and cell death: outer membrane permeabilization and beyond. Nature Reviews Molecular Cell Biology 11 621-632. (doi:10.1038/nrm2952)

Tsui KH, Lin LT, Horng HC, Chang R, Huang BS, Cheng JT \& Wang PH 2014 Gene expression of cumulus cells in women with poor ovarian response after dehydroepiandrosterone supplementation. Taiwanese Journal of Obstetrics and Gynecology 53 559-565. (doi:10.1016/j. tjog.2014.09.003)
Van Blerkom J 2011 Mitochondrial function in the human oocyte and embryo and their role in developmental competence. Mitochondrion 11 797-813. (doi:10.1016/j.mito.2010.09.012)

Vanden Berghe T, Vanlangenakker N, Parthoens E, Deckers W, Devos M, Festjens N, Guerin CJ, Brunk UT, Declercq W \& Vandenabeele P 2010 Necroptosis, necrosis and secondary necrosis converge on similar cellular disintegration features. Cell Death and Differentiation $17922-$ 930. (doi:10.1038/cdd.2009.184)

Vandenabeele P, Galluzzi L, Vanden Berghe T \& Kroemer G 2010 Molecular mechanisms of necroptosis: an ordered cellular explosion. Nature Reviews Molecular Cell Biology 11 700-714. (doi:10.1038/ nrm2970)

Vermes I, Haanen C, Steffens-Nakken H \& Reutelingsperger C 1995 A novel assay for apoptosis. Flow cytometric detection of phosphatidylserine expression on early apoptotic cells using fluorescein labelled Annexin V. Journal of Immunological Methods 184 39-51. (doi:10.1016/00221759(95)00072-I)

Wathlet S, Adriaenssens T, Segers I, Verheyen G, Janssens R, Coucke W, Devroey P \& Smitz J 2012 New candidate genes to predict pregnancy outcome in single embryo transfer cycles when using cumulus cell gene expression. Fertility and Sterility 98 432-439.e431-e434. (doi:10.1016/j. fertnstert.2012.05.007)

Wu W, Liu P \& Li J 2012 Necroptosis: an emerging form of programmed cell death. Critical Reviews in Oncology/Hematology 82 249-258. (doi:10.1016/j.critrevonc.2011.08.004)

Xiong S, Mu T, Wang G \& Jiang X 2014 Mitochondria-mediated apoptosis in mammals. Protein and Cell 5 737-749. (doi:10.1007/s13238-0140089-1)

Zeng HT, Ren Z, Yeung WS, Shu YM, Xu YW, Zhuang GL \& Liang XY 2007 Low mitochondrial DNA and ATP contents contribute to the absence of birefringent spindle imaged with PolScope in in vitro matured human oocytes. Human Reproduction 22 1681-1686. (doi:10.1093/humrep/ dem070)

Received 8 January 2017

First decision 2 February 2017

Revised manuscript received 6 may 2017

Accepted 11 May 2017 